\title{
Model experiments and direct stability assessments on pure loss of stability in stern quartering waves
}

\author{
Jiang, Lu, China Ship Scientific Research Center (currently visiting at University of Strathclyde) \\ lujiang1980@aliyun.com \\ Min, Gu, China Ship Scientific Research Center gumin702@163.com \\ Evangelos Boulougouris, Maritime Safety Research Centre, University of Strathclyde, \\ evangelos.boulougouris@strath.ac.uk
}

\begin{abstract}
The criteria for direct stability assessment of pure loss of stability are currently under development at IMO for second generation intact stability criteria. For providing a mathematical model of pure loss of stability with sufficient accuracy and practically useful, firstly, a surge-sway-roll-yaw coupled mathematical model is reestablished with unified expressions based on an MMG standard method for ship maneuvering predictions and existing mathematical models for broaching and parametric rolling predictions. Secondly, model tests are carried out in stern quartering waves. Thirdly, the effect of some crucial terms, such as the higher-order coefficients, the maneuvering motions, diffraction forces, heel-induced hydrodynamic forces on predicting pure loss of stability are studied. Finally, the types of roll motions are identified during pure loss of stability. The result shows the 4 DOF surge-roll-sway-yaw coupled motion can be utilized for direct stability assessment of pure loss of stability in stern quartering waves.
\end{abstract}

Keywords: Pure loss of stability; second generation intact stability criteria; ONR tumblehome; stability in waves; IMO

\section{LIST OF SYMBOLS}

$a_{H} \quad$ Rudder force increase factor

$A E, F E$ After section and forward section

$A_{R} \quad$ Rudder area

$A_{R P}, A_{R S}$ The port and starboard rudder area

$B(x)$ Sectional breadth

$C_{T} \quad$ Total resistance coefficient in calm water

$d \quad$ Ship draft

$d(x)$ Sectional draught

$D_{P} \quad$ Propeller diameter

$D(p)$ Roll damping moment

$F_{N} \quad$ Rudder normal force

$F_{n} \quad$ Froude number based on ship length

$f_{\alpha} \quad$ Rudder lifting slope coefficient

$g$ Gravitational acceleration

GM Metacentric height

$G Z_{W}$ Righting arm in waves

$H_{R} \quad$ Rudder span length

$I_{x x}, J_{x x}$ Moment and added moment of inertia in roll

$I_{z z}, J_{z z}$ Moment and added moment of inertia in yaw
$J_{p}$ Propeller advanced ratio

$k$ Wave number

$K_{r}, N_{r}, Y_{r}$ The derivative of roll moment, yaw moment and sway force corresponding to yaw rate, their nondimensional are $K_{r}^{\prime}, N_{r}^{\prime}, Y_{r}^{\prime}$

$K_{r r r}, N_{r r r}, Y_{r r r}$ The derivative of roll moment, yaw moment and sway force corresponding to cubic yaw rate, their nondimensional are $K_{r r r}^{\prime}, N_{r r r}^{\prime}, Y_{r r}^{\prime}$

$K_{r|\phi|}, N_{r|\phi|}, Y_{r|\varphi|}$ The derivative of roll moment, yaw moment and sway force corresponding to yaw rate and heeling angle, their nondimensional are $K_{r|\phi|}^{\prime}, N_{r|\phi|}^{\prime}, Y_{r|\phi|}^{\prime}$

$K_{v r r}, N_{v r r}, Y_{v r r}$ The derivative of roll moment, yaw moment and sway force corresponding to squared yaw rate and sway velocity, their nondimensional are $K_{v r r}^{\prime}, N_{v r}^{\prime}, Y_{v r r}^{\prime}$

$K_{v v}, N_{v v}, Y_{v v r}$ The derivative of roll moment, yaw moment and sway force corresponding to 
squared yaw rate and sway velocity, their nondimensional $K_{v v}^{\prime}, N_{v v r}^{\prime}, Y_{v v r}^{\prime}$

$K_{v}, N_{v}, Y_{v}$ The derivative of roll moment, yaw moment and sway force corresponding to sway velocity, their nondimensional $K_{v}^{\prime}, N_{v}^{\prime}, Y_{v}^{\prime}$

$K_{v v}, N_{v v}, Y_{v v}$ The derivative of roll moment, yaw moment and sway force corresponding to cubic sway velocity, their nondimensional $K_{v v v}^{\prime}, N_{v v v}^{\prime}, Y_{v v v}^{\prime}$

$K_{v|q|}, N_{v \mid q}, Y_{v|q|}$ The derivative of roll moment, yaw moment and sway force corresponding to sway velocity and heeling angle, their nondimensional $K_{v \mid \varphi}^{\prime}, N_{v|\varphi|}^{\prime}, Y_{v|\varphi|}^{\prime}$

$K_{\phi}, N_{\phi}, Y_{\phi}$ The derivative of roll moment, yaw moment and sway force corresponding to roll angle, their nondimensional $K_{\varphi}^{\prime}, N_{\varphi}^{\prime}, Y_{\varphi}^{\prime}$

$K_{P}$ Rudder gain

$K_{T}$ Thrust coefficient of the propeller

$L_{P P}$ Ship length between perpendiculars

$\ell_{R}$ ' Correction factor for flow-straightening due to yaw

$m \quad$ Ship mass

$m_{x}, m_{y}$ Added mass in surge and sway

$n_{P}$ Propeller revolution number

$O G$ The vertical distance between the center of gravity and waterline

$p$ Roll rate

$r \quad$ Yaw rate

$R \quad$ Ship resistance

$S(x)$ Sectional area

$S_{y}(x)$ Added mass of one section at sway direction

$S_{y} l_{\eta}(x)$ Added moment of one section at roll direction

$S_{F} \quad$ Wetted hull surface area

$t_{P} \quad$ Thrust deduction factor

$t_{R} \quad$ Steering resistance deduction factor

$T$ Propeller thrust

$T_{E} \quad$ The time constant for steering gear

$T_{D} \quad$ The time constant for differential control

$T_{\varphi} \quad$ Natural roll period

$u, v$ Surge and sway velocity

$u_{R}$ Longitudinal inflow velocity component to the rudder

$U \quad$ Ship forward velocity

$w_{P} \quad$ Wake fraction at propeller position

$w_{R} \quad$ Wake fraction at rudder position
W Ship weight

$x_{H R}$ The longitudinal position of additional sway force due to the rudder

$x_{R} \quad$ The longitudinal position of the rudder

$X_{H}, Y_{H}, N_{H}, K_{H}$ Surge force, sway force, yaw moment and roll moment around center of ship gravity acting on ship hull

$X_{p} \quad$ Surge force due to the propeller

$X_{R}, Y_{R}, N_{R}, K_{R}$ Surge force, sway force, yaw moment and roll moment around center of ship gravity by steering

$X_{r r}$ The derivative of surge force corresponding to squared yaw rate, its nondimensional is $X_{r r}^{\prime}$

$X_{v r}$ The derivative of surge force corresponding to sway velocity and yaw rate, its nondimensional is $X_{v r}^{\prime}$

$X_{v v}$ The derivative of surge force corresponding to squared sway velocity, its nondimensional is $X_{v v}^{\prime}$

$X_{v v v}$ The derivative of surge force corresponding to 4 th order sway velocity, its nondimensional is $X_{v w v}^{\prime}$

$X_{w}, Y_{W}, N_{W}, K_{W}$ Surge force, sway force, yaw moment and roll moment around center of ship gravity acting on ship hull induced by waves

$Z_{H}$ The vertical position of the center of sway force due to sway motion

$z_{H R}$ The vertical position of additional sway force due to the rudder

$z_{R} \quad$ The vertical position of the center of the rudder

$\alpha$ The linear roll damping coefficient

$\alpha_{R} \quad$ Effective inflow angle to the rudder

$\beta \quad$ Hull drift angle

$\delta \quad$ Rudder angle

$\eta \quad$ The ratio of propeller diameter to rudder span

$\varepsilon \quad$ The ratio of wake fraction at propeller and rudder position

$\kappa \quad$ Propeller-induced flow velocity factor

$\lambda$ Wave length

$\Lambda$ Ruder aspect ratio

$\varphi \quad$ Roll angle

$\gamma$ The cubic nonlinear roll damping coefficient

$\gamma_{R} \quad$ Flow-straightening effect coefficient

$\theta \quad$ Pitch angle

$\chi$ Yaw angle from wave direction

$\chi_{c} \quad$ Yaw angle of autopilot course

$\rho$ Water density 
$\omega \quad$ Wave frequency

$\omega_{e}$ Averaged encounter frequency

$\xi_{G}$ The longitudinal position of the center of ship gravity from a wave trough

$\left(\xi_{G}, \eta_{G}, \zeta_{G}\right)$ Position of center of ship gravity in the space-fixed coordinate system

$\zeta_{w}$ Wave amplitude

\section{INTRODUCTION}

The guidelines for direct stability assessment of five stability failure models including pure loss of stability are under development at the International Maritime Organization (IMO) for the second generation intact stability criteria (IMO SDC 4, 2017). Once the crest of the large wave passes the midship section of a ship with a slightly higher speed than ship speed, the state of stability loss at the crest may exist long enough to evolve a large heel angle, or even capsizing. Level 1 and 2 are related to GM and GZ in waves, and direct stability assessment is related to the time domain simulation with hydrodynamics, stochastic, statistics, probability and so on. Time-domain simulation is a key step for direct stability assessment. It is urgently required to establish a standard mathematical model or a guideline that is sufficiently accurate and practically useful for direct stability assessment of pure loss of stability.

\section{BACKGROUND}

The capsize angle to leeward side is defined as the minimum between the angle of vanishing stability and the critical angle. The capsize angle to windward side is defined as the maximum between the angle of vanishing stability and the critical angle. The critical angle is usually considered as the minimum between a large angle of roll (e.g. $50 \mathrm{deg}$ ) and the angle of progressive flooding. (IMO SDC1, 2014). Here, the capsizing means the ship turns over in the water and the results shown in this paper are cut at 70 degrees.

The stability in waves often becomes larger at the trough and becomes smaller at the crest comparing with that in calm water (Paulling, 1961). Pure loss of stability was identified during the model experiments in San-Francisco Bay (Paulling, et al., 1972, 1975), and considered as a static capsizing mode that ship loses static restoring in waves (Okakley et al, 1974). Many researchers focused on the method to calculate the GZ curve in regular and irregular waves (Kuo et al., 1986; Hamamoto \& Nomoto, 1982). One method for calculating the restoring variation in waves was developed by using the strip method of heave and pitch motion instead of static method (Umeda et al., 2005) The method is further utilized using time-varied amplitude and phase of heave and pitch for direct stability assessment of pure loss of stability in following waves by the Lu et al. (2019).

The restoring moment in irregular waves is considered a stochastic process (Dunwoody, 1989; Palmquist, 1994; Bulian \& Francescutto, 2006). The Grim effective wave is used to evaluate the probability of capsizing due to pure-loss of stability in short-crested irregular stern-quartering waves (Umeda \& Yamakoshi, 1993). The waves are assumed to be a narrow-banded stochastic process instead of the Grim effective wave (Vermeer, 1990). The concept of a "critical wave" is used to evaluate the probability of capsizing (Themelis \& Spyrou, 2007). The effective wave approach combined with the uncrossing theory is used to produce a timedependent probability of stability failure (Bulian et al., 2008). The application of the split-time method to pure loss of stability is discussed by Belenky et al. (2015).

Without external heel moment, once the wave crest passes the ship, the ship will finally return to the upright position with regained stability except for cases that the ship already heel too far or the metacentric height in the wave is negative. (IMO SDC 3, 2016). Hashimoto carried out experiments on pure loss of stability in following seas with an initial heel moment induced by cargo shift (Hashimoto, 2009). The authors further confirmed capsizing due to pure loss of stability may not be reproduced in the simulation and experiment in following seas without initial heel moments ( $\mathrm{Lu}$ et al., 2019). Umeda firstly pointed out that pure loss of stability in stern quartering waves could not be really pure, and also the heeling moment induced by a centrifugal force due to ship maneuvering motions are the relevant external moments (Kubo et al., 2012). Umeda further agued the capsizing of the actual ship in stern quartering waves could due to ship maneuvering motions of sway and yaw (Umeda et al., 2019).

Pure loss of stability in astern waves is a nonlinear phenomenon involving large amplitude roll motion, and the loss of stability is also related 
periodic motion, accumulative broaching and so on (Spyrou, 1997), and it is still difficult to be predicted quantitatively. Bassler et al. (2011) argued that the difficulty of developing criteria for pure-loss of stability is not limited to the calculation of the GZ curve in waves. Neves (2016) reviewed the latest methods for predicting the occurrence of pure loss of stability. Japanese delegation (IMO SLF55, 2013) notes that predicting pure loss of stability with their newly-developed 4 degrees of freedom (DOF) mathematical model (Kubo et al., 2012) is more accurate than the 2 DOF mathematical model (Hashimoto, 2009). The delegations for the second generation intact stability criteria at IMO SDC 4 gave top priority to discussing the guidelines for direct stability assessment and the use of the 4 DOF for predicting pure loss of stability has been agreed at the current stage (IMO SDC 4, 2017).

Though the 4 DOF mathematical model for predicting pure loss of stability has not been investigated widely with simulations and experiments, a 4 DOF mathematical model for broaching prediction (Umeda, 1999) has been investigated for many years. For providing an accurate mathematical model for broaching prediction, Umeda and Hashimoto had investigated essential terms in the 4 DOF mathematical model one by one by utilizing fishing vessels. Nonlinear maneuvering forces in calm water (Umeda \& Hashimoto, 2002), wave effect on linear maneuvering forces, roll restoring and rudder force (Umeda et al., 2003), and several nonlinear factors were also investigated, such as nonlinear wave forces, nonlinear sway-yaw coupling, wave effect on propeller thrust, heel-induced hydrodynamic forces for large heel angle in calm water (Hashimoto et al., 2004), and wave effect on heel-induced hydrodynamic forces for large heel angle. A simplified mathematical model was proposed for practical uses (Hashimoto et al., 2011a). Existing 4 DOF mathematical model was used for broaching prediction of the ONR tumblehome vessel, and a fair quantitative prediction was realized (Hashimoto et al., 2011b). Broaching is a nonlinear phenomenon related to the ship maneuvering in the wave, and above 4 DOF mathematical models are based on a Maneuvering Modeling Group (MMG) model, but simulation methods without standard expressions could not be used in general. Therefore, an MMG standard method for ship maneuvering predictions was introduced (Yasukawa \& Yoshimura, 2015). A 4 DOF mathematical model was refined for broaching prediction of the ONR flare topside vessel (Umeda et al., 2016).

For drafting guidelines for direct stability assessment, several crucial elements for predicting parametric roll were investigated with simulations and experiments (Lu et al., 2017), and some crucial terms in the 4 DOF mathematical for predicting pure loss of stability still require further experimental and numerical studies with more examples. The physical mechanism of pure loss of stability is different from that of broaching and a 4 DOF standard mathematical model for predicting pure loss of stability has not been established widely. Therefore, systematic studies on the 4 DOF mathematical model for direct stability assessment for pure loss of stability are hot tasks at this stage. Also, IMO is calling for the validation of numerical methods or guidelines for the finalization of second generation intact stability with examples.

Based on the MMG standard method and existing mathematical model for broaching, pure loss of stability and parametric rolling, the authors tried to provide a 4 DOF standard mathematical model [ $\mathrm{Lu}$ et al, 2017, 2018]. The present study intends to provide a 4 DOF mathematical models for pure loss of stability in stern quartering waves. Some crucial terms in the mathematical models were investigated and a tumblehome vessel which is provided by an IMO's intersessional correspondence group as one of the standard ships for developing the second generation intact stability criteria is used. The tumblehome vessel also could prone to suffer stability failure in severe waves (Bassler et al., 2007, Galbraith\& Boulougouris, 2015).

\section{MATHEMATICAL MODEL}

\subsection{Coordinate systems}

A space-fixed coordinate system $O-\xi \eta \zeta$ with the origin at a wave trough, a body-fixed system $G-x^{\prime} y^{\prime} z^{\prime}$ with the origin at the center of gravity of the ship, and a horizontal body coordinate system(Hamamoto\& Kim, 1993) $G-x y z$ which has the same origin with the body-fixed system but does not rotated around the $\mathrm{x}$-axis and $\mathrm{y}$-axis are adopted as shown in Fig.1. 


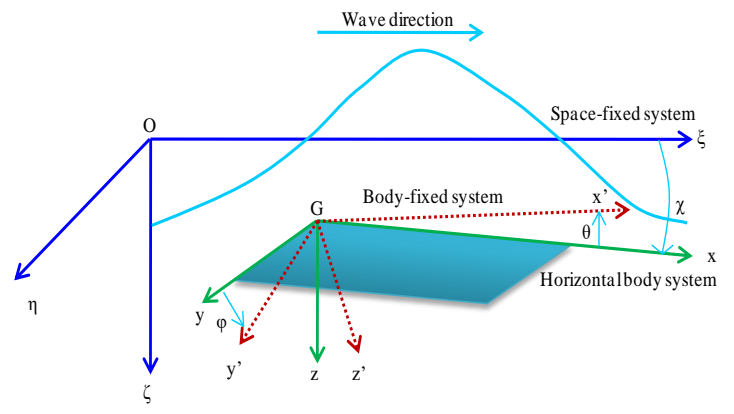

Figure 1: Coordinate systems

The relationships between the horizontal body coordinate system $G-x y z$, the body-fixed system $G-x^{\prime} y^{\prime} z^{\prime}$ and the space-fixed system $O-\xi \eta \zeta$ are shown in Eq. (1) and Eq. (2), respectively.

$$
\left[\begin{array}{l}
x \\
y \\
z
\end{array}\right]=\left[\begin{array}{lcc}
\cos \theta & \sin \phi \sin \theta & \cos \phi \sin \theta \\
0 & \cos \varphi & -\sin \varphi \\
-\sin \theta & \sin \varphi \cos \theta & \cos \varphi \cos \theta
\end{array}\right]\left[\begin{array}{l}
x^{\prime} \\
y^{\prime} \\
z^{\prime}
\end{array}\right]
$$

$$
\left[\begin{array}{c}
\xi-\xi_{G} \\
\eta-\eta_{G} \\
\zeta-\zeta_{G}
\end{array}\right]=\left[\begin{array}{ccc}
\cos \theta \cos \chi & \sin \varphi \sin \theta \cos \chi & \cos \varphi \sin \theta \cos \chi \\
-\cos \varphi \sin \chi & +\sin \varphi \sin \chi \\
\cos \theta \sin \chi & \sin \varphi \sin \theta \sin \chi & \cos \varphi \sin \theta \sin \chi \\
-\sin \theta & \sin \varphi \cos \theta & -\sin \varphi \cos \chi \\
y^{\prime}
\end{array}\right]\left[\begin{array}{l}
x^{\prime} \\
\\
z^{\prime}
\end{array}\right]
$$

\subsection{Mathematical model}

Heave and pitch response could be treated dynamically or quasi-statically depending on the encounter frequency. In the case of astern waves, the encounter frequency is much lower than the natural frequencies of heave and pitch so that coupling with heave and pitch can be approximated by their stable equilibria (Matsuda \& Umeda, 1997). The 4 DOF mathematical model is expressed by the surge, sway, yaw and roll motions referring to the references (Yasukawa \& Yoshimura, 2015; Umeda et al., 2016) as shown in Eq. (3) to Eq. (6), respectively. The control equation for keeping course by steering is added in the 4 DOF mathematical model as shown in Eq. (7).

$$
\begin{array}{r}
\left(m+m_{x}\right) \dot{u}-\left(m+m_{y}\right) v r=X_{H}+X_{R}+X_{P}+X_{W} \\
\left(m+m_{y}\right) \dot{v}+\left(m+m_{x}\right) u r=Y_{H}+Y_{R}+Y_{W} \\
\left(I_{z z}+J_{z z}\right) \dot{r}=N_{H}+N_{R}+N_{W}
\end{array}
$$

$$
\begin{aligned}
& \left(I_{x x}+J_{x x}\right) \dot{p}-m_{x} z_{H} u r-m_{y} z_{H} \dot{v}=K_{H}+K_{R}+K_{W_{-} D l f} \\
& -D(\dot{\varphi})-W G Z_{W_{-} F K}\left(\xi_{G} / \lambda, \chi, \varphi\right) \\
& \quad \dot{\delta}=\left\{-\delta-K_{P}\left(\chi-\chi_{C}\right)-K_{P} T_{D} r\right\} / T_{E}
\end{aligned}
$$

The subscript H, R, P, and W refer to hull, rudder, propeller, and wave, respectively.

\subsection{Hydrodynamic forces acting on ship hull}

Hydrodynamic forces acting on a ship hull of an MMG standard method (Yasukawa \& Yoshimura, 2015 ) is referenced with the roll motion and heelinduced hydrodynamic forces taken into account.

The hull forces in still water $X_{H}, Y_{H}, N_{H}$ and $K_{H}$ are expressed as follows referring to the references (Yasukawa \& Yoshimura, 2015; Umeda et al., 2016):

$$
\begin{aligned}
X_{H}= & -R(u)+\frac{1}{2} \rho L_{p p} d U^{2}\left(X_{v v}^{\prime} \cdot v^{2}+X_{v r}^{\prime} \cdot v^{\prime} r^{\prime}\right. \\
+ & \left.X_{r r}^{\prime} \cdot r^{\prime 2}+X_{v v v}^{\prime} \cdot v^{\prime 4}\right) \\
Y_{H}= & \frac{1}{2} \rho L_{p p} d U^{2}\left(Y_{v}^{\prime} \cdot v^{\prime}+Y_{r}^{\prime} \cdot r^{\prime}\right. \\
& +Y_{v v v}^{\prime} \cdot v^{\prime 3}+Y_{v v r}^{\prime} \cdot v^{\prime 2} r^{\prime}+Y_{v r r}^{\prime} \cdot v^{\prime} r^{\prime 2}+Y_{r r r}^{\prime} \cdot r^{\prime 3} \\
& \left.+Y_{\varphi}^{\prime} \cdot \varphi+Y_{v|\varphi|}^{\prime} \cdot v^{\prime}|\varphi|+Y_{r|\varphi|}^{\prime} \cdot r^{\prime}|\varphi|\right) \\
N_{H}= & \frac{1}{2} \rho L_{p p}^{2} d U^{2}\left(N_{v}^{\prime} \cdot v^{\prime}+N_{r}^{\prime} \cdot r^{\prime}\right. \\
& +N_{v v v}^{\prime} \cdot v^{\prime 3}+N_{v v r}^{\prime} \cdot v^{\prime 2} r^{\prime}+N_{v r r}^{\prime} \cdot v^{\prime} r^{\prime 2}+N_{r r r}^{\prime} \cdot r^{33} \\
& \left.+N_{\varphi}^{\prime} \cdot \varphi+N_{v|\varphi|}^{\prime} \cdot v^{\prime}|\varphi|+N_{r|\varphi|}^{\prime} \cdot r^{\prime}|\varphi|\right) \\
K_{H}= & \frac{1}{2} \rho L_{p p} d^{2} U^{2}\left(K_{v}^{\prime} \cdot v^{\prime}+K_{r}^{\prime} \cdot r^{\prime}\right. \\
& +K_{v v v}^{\prime} \cdot v^{\prime 3}+K_{v v r}^{\prime} \cdot v^{\prime 2} r^{\prime}+K_{v r r}^{\prime} \cdot v^{\prime} r^{2}+K_{r r r}^{\prime} \cdot r^{\prime 3} \\
& \left.+K_{\phi}^{\prime} \cdot \phi+K_{v|\varphi|}^{\prime} \cdot v^{\prime}|\varphi|+K_{r|\varphi|}^{\prime} \cdot r^{\prime}|\varphi|\right) \\
= & -Y_{H} \times Z_{H}
\end{aligned}
$$

where $v^{\prime}, r^{\prime}$ denote nondimensional sway velocity, and yaw rate, respectively and are expressed as follows:

$$
v^{\prime}=\frac{v}{U} \quad, r^{\prime}=\frac{r L_{p p}}{U}
$$

Each maneuvering coefficient can be determined by a circular motion test, or oblique towing test (OTT). For providing unified expressions, the nondimensional maneuvering coefficients are rewritten as follows: 


$$
\begin{aligned}
& X_{v v}^{\prime}=\frac{X_{v v}}{\frac{1}{2} \rho L_{P P} d} \quad, X_{v r}^{\prime}=\frac{X_{v r}}{\frac{1}{2} \rho L_{P P}^{2} d} \\
& X_{r r}^{\prime}=\frac{X_{r r}}{\frac{1}{2} \rho L_{P P}^{3} d}, X_{v v v v}^{\prime}=\frac{X_{v v v v}}{\frac{1}{2} \rho L_{P P} d / U^{2}} \\
& Y_{v}^{\prime}=\frac{Y_{v}}{\frac{1}{2} \rho L_{P P} d U}, Y_{r}^{\prime}=\frac{Y_{r}}{\frac{1}{2} \rho L_{P P}^{2} d U}, Y_{\varphi}^{\prime}=\frac{Y_{\varphi}}{\frac{1}{2} \rho L_{P P} d U^{2}} \\
& Y_{v v}^{\prime}=\frac{Y_{v v v}}{\frac{1}{2} \rho L_{P P} d / U}, Y_{v v r}^{\prime}=\frac{Y_{v v}}{\frac{1}{2} \rho L_{P P}^{2} d / U}, Y_{v r r}^{\prime}=\frac{Y_{v r r}}{\frac{1}{2} \rho L_{P P}^{3} d / U} \\
& Y_{r r r}^{\prime}=\frac{Y_{r r r}}{\frac{1}{2} \rho L_{P P}^{4} d / U}, Y_{v|q|}^{\prime}=\frac{Y_{v|q|}}{\frac{1}{2} \rho L_{P P} d U}, Y_{r|q|}^{\prime}=\frac{Y_{r|q|}}{\frac{1}{2} \rho L_{P P}^{2} d U} \\
& N_{v}^{\prime}=\frac{N_{v}}{\frac{1}{2} \rho L_{P P}^{2} d U}, N_{r}^{\prime}=\frac{N_{r}}{\frac{1}{2} \rho L_{P P}^{3} d U}, N_{\varphi}^{\prime}=\frac{N_{\varphi}}{\frac{1}{2} \rho L_{P P}^{2} d U^{2}} \\
& N_{v v}^{\prime}=\frac{N_{v v v}}{\frac{1}{2} \rho L_{P P}^{2} d / U}, N_{v v r}^{\prime}=\frac{N_{v v r}}{\frac{1}{2} \rho L_{P P}^{3} d / U}, N_{v r r}^{\prime}=\frac{N_{v r}}{\frac{1}{2} \rho L_{P P}^{4} d / U} \\
& N_{r r}^{\prime}=\frac{N_{r r}}{\frac{1}{2} \rho L_{P P}^{5} d / U}, N_{v|q|}^{\prime}=\frac{N_{v|q|}}{\frac{1}{2} \rho L_{P P}^{2} d U}, N_{r|q|}^{\prime}=\frac{N_{r|\phi|}}{\frac{1}{2} \rho L_{P P}^{3} d U} \\
& K_{v}^{\prime}=\frac{K_{v}}{\frac{1}{2} \rho L_{P P} d^{2} U}, K_{r}^{\prime}=\frac{K_{r}}{\frac{1}{2} \rho L_{P P}^{2} d^{2} U}, K_{\varphi}^{\prime}=\frac{K_{\varphi}}{\frac{1}{2} \rho L_{P P} d^{2} U^{2}} \\
& K_{v v}^{\prime}=\frac{K_{v v}}{\frac{1}{2} \rho L_{P P} d^{2} / U}, K_{v r r}^{\prime}=\frac{K_{v r}}{\frac{1}{2} \rho L_{P P}^{3} d^{2} / U}, K_{r r}^{\prime}=\frac{K_{r r}}{\frac{1}{2} \rho L_{P P}^{4} d^{2} / U}
\end{aligned}
$$

\subsection{Propeller thrust and the hull resistance in still water}

The surging force due to propeller thrust $X_{P}$ with twin propellers is expressed as follows:

$$
\begin{aligned}
& X_{P}=2 \times\left(1-t_{P}\right) T \\
& T=\rho n_{P}^{2} D_{P}^{4} K_{T}\left(J_{P}\right) \\
& J_{P}=\frac{\left(1-w_{P}\right) u}{n_{P} D_{P}}
\end{aligned}
$$

The hull resistance in still water $R$ in the surge motion is expressed as follows:

$$
R=\frac{1}{2} \rho S_{F} u^{2} C_{T}\left(\frac{u}{\sqrt{g L_{P P}}}\right)
$$

\subsection{Hydrodynamic force by steering}

Hydrodynamic forces acting on a ship hull of an MMG standard method (Yasukawa \& Yoshimura, 2015) are referenced.
The steering rudder forces components $X_{R}, Y_{R}, N_{R}$ and $K_{R}$ with twin rudders (Khanfir, et al., 2011) are referred and expressed as follows(S: starboard; P: port). The average values of $\overline{\gamma_{R}}, \overline{\ell_{R}^{\prime}}$ are used in this paper.

$$
\begin{aligned}
& X_{R}=-\left(1-t_{R}\right) F_{N} \sin \delta \\
& Y_{R}=-\left(1+a_{H}\right) F_{N} \cos \delta \\
& N_{R}=-\left(x_{R}+a_{H} x_{H R}\right) F_{N} \cos \delta \\
& K_{R}=\left(z_{R}+a_{H} z_{H R}\right) F_{N} \cos \delta
\end{aligned}
$$

where

$$
\begin{gathered}
F_{N}=\frac{1}{2} \rho\left(A_{R(S)}+A_{R(P)}\right) u_{R}^{2} f_{\alpha} \sin \alpha_{R} \\
u_{R}=\varepsilon\left(1-w_{p}\right) u \sqrt{\eta\left\{1+\kappa\left(\sqrt{1+\frac{8 K_{T}\left(J_{P}\right)}{\pi J_{P}^{2}}}-1\right)\right\}^{2}+1-\eta} \\
\alpha_{R}=\delta-\overline{\gamma_{R}} \frac{U}{u_{R}}\left(\beta-\overline{\ell_{R}^{\prime}} r^{\prime}\right) \\
\overline{\gamma_{R}}=\frac{1}{2}\left(\gamma_{R(s)}+\gamma_{R(P)}\right) \\
\overline{\ell_{R}^{-}}=\frac{1}{2}\left(\ell_{R(s)}^{\prime}+\ell_{R(P)}^{\prime}\right) \\
f_{\alpha}=\frac{6.13 \Lambda}{2.25+\Lambda}, \varepsilon=\frac{1-w_{R}}{1-w_{P}} \\
\eta=\frac{D_{P}}{H_{R}}, \beta=\arctan \left(\frac{-v}{u}\right), U=\sqrt{u^{2}+v^{2}}
\end{gathered}
$$

\subsection{Excited wave force}

The wave-induced forces including the FroudeKrylov force (W_FK), the diffraction force (W_Dif), and hydrodynamic lift forces acting on the hull are rewritten as follows referring to the reference (Umeda \& Hashimoto, 2002). The Froude-Krylov roll moment is taken into account for calculating the roll restoring variation so that only the diffraction force is used in Eq. (39) for avoiding double counting of the Froude-Krylov roll moment in case of oblique waves.

$$
\begin{aligned}
& X_{W}\left(\xi_{G} / \lambda, u, \chi\right)=X_{W_{-} F K}\left(\xi_{G} / \lambda, u, \chi\right) \\
& =-\rho g \zeta_{w} k \cos \chi \int_{A E}^{F E} C_{1}(x) S(x) e^{-k d(x) / 2} \sin k\left(\xi_{G}+x \cos \chi\right) d x \\
& Y_{W}\left(\xi_{G} / \lambda, u, \chi\right)=Y_{W_{-} F K}\left(\xi_{G} / \lambda, u, \chi\right)+Y_{W_{-} D i f f}\left(\xi_{G} / \lambda, u, \chi\right) \\
& =\rho g \zeta_{w} k \sin \chi \int_{A E}^{F E} C_{1}(x) S(x) e^{-k d(x) / 2} \sin k\left(\xi_{G}+x \cos \chi\right) d x \\
& +\zeta_{w} \omega \omega_{e} \sin \chi \int_{A E}^{F E} \rho S_{y}(x) e^{-k d(x) / 2} \sin k\left(\xi_{G}+x \cos \chi\right) d x \\
& -\zeta_{w} \omega u \sin \chi\left[\rho S_{y}(x) e^{-k d(x) / 2} \cos k\left(\xi_{G}+x \cos \chi\right)\right]_{A E}^{F E}
\end{aligned}
$$




$$
\begin{aligned}
& N_{W}\left(\xi_{G} / \lambda, \chi\right)=N_{W_{-} F K}\left(\xi_{G} / \lambda, u, \chi\right)+N_{W_{-} D i f f}\left(\xi_{G} / \lambda, u, \chi\right) \\
& =\rho g \zeta_{w} k \sin \chi \int_{A E}^{F E} C_{1}(x) S(x) e^{-k d(x) / 2} x \sin k\left(\xi_{G}+x \cos \chi\right) d x \\
& +\zeta_{w} \omega \omega_{e} \sin \chi \int_{A E}^{F E} \rho S_{y}(x) e^{-k d(x) / 2} x \sin k\left(\xi_{G}+x \cos \chi\right) d x \\
& +\zeta_{w} \omega u \sin \chi \int_{A E}^{F E} \rho S_{y}(x) e^{-k d(x) / 2} \cos k\left(\xi_{G}+x \cos \chi\right) d x \\
& -\zeta_{w} \omega u \sin \chi\left[\rho S_{y}(x) e^{-k d(x) / 2} x \cos k\left(\xi_{G}+x \cos \chi\right)\right]_{A E}^{F E} \\
& K_{W}\left(\xi_{G} / \lambda, u, \chi\right)=K_{W_{-} F K}\left(\xi_{G} / \lambda, u, \chi\right)+K_{W_{-} D i f f}\left(\xi_{G} / \lambda, u, \chi\right) \\
& =-\rho g \zeta_{w} k \sin \chi \int_{A E}^{F E} C_{1}(x) \frac{B(x)}{2}\{d(x)\}^{2} e^{-k d(x) / 2} \sin k\left(\xi_{G}+x \cos \chi\right) d x \\
& -\rho g \zeta_{w} k^{2} \sin \chi \int_{A E}^{F E} C_{4}(x)\left\{\frac{B(x)}{2}\right\}^{3} d(x) e^{-k d(x) / 2} \sin k\left(\xi_{G}+x \cos \chi\right) d x \\
& -\zeta_{w} \omega \omega_{e} \sin \chi \int_{A E}^{F E} \rho S_{y} l_{\eta}(x) e^{-k d(x) / 2} \sin k\left(\xi_{G}+x \cos \chi\right) d x \\
& +\zeta_{w} \omega u \sin \chi\left[\rho S_{y} l_{\eta}(x) e^{-k d(x) / 2} \cos k\left(\xi_{G}+x \cos \chi\right)\right]_{A E}^{F E} \\
& +Y_{w}\left(\xi_{G} / \lambda, u, \chi\right) \cdot \overline{O G} \\
& C_{1}=\frac{\sin (k \sin \chi \cdot B(x) / 2)}{k \sin \chi \cdot B(x) / 2} \\
& C_{4}=\{k \sin \chi \cdot B(x) / 2\}^{-3}[2 \sin \{k \sin \chi \cdot B(x) / 2\} \\
& \quad-k \sin \chi \cdot B(x) \cos \{k \sin \chi \cdot B(x) / 2\}]
\end{aligned}
$$

\subsection{Roll restoring force variation}

Pure loss of stability is one of the problems related to the roll restoring force variation. The restoring force variation in oblique waves can be calculated by integrating the pressure around the instantaneously wetted hull surface with a static balance of heave and pitch as shown in Eq.(42) which is based on Froude-Krylov assumption and utilized to predict parametric rolling(Lu et al., 2017). The Froude-Krylov roll moment is taken into account in Eq. (42) considering oblique wave heading in this paper, but the effect of wave heading is converted into the change of the effective wave height in longitudinal waves by using Grim's effective wave concept in the references (Umeda \&Yamakoshi, 1994; Kubo et al., 2012).

$$
\begin{array}{r}
W \cdot G Z_{W-F K}=\rho g \int_{A E}^{F E} y\left(x, \xi_{G} / \lambda\right) \cdot A\left(x, \xi_{G} / \lambda\right) d x+\rho g \sin \chi \cdot \\
\int_{A E}^{F E} z\left(x, \xi_{G} / \lambda\right) \cdot F(x) \cdot A\left(x, \xi_{G} / \lambda\right) \cdot \sin \left(\xi_{G}+x \cos \chi\right) d x \\
F(x)=\zeta_{w} k \frac{\sin \left(k \frac{B(x)}{2} \sin \chi\right)}{k \frac{B(x)}{2} \sin \chi} e^{-k d(x)}(43)
\end{array}
$$

where, $A\left(x, \xi_{G} / \lambda\right)$ is the submerged area of a local section of the ship. $y\left(x, \xi_{G} / \lambda\right)$ is the transverse position of the buoyancy center of a local section. $z\left(x, \xi_{G} / \lambda\right)$ is the vertical position of the buoyancy center of a local section.

\subsection{Roll damping force}

Roll damping is one of the essential terms for predicting roll motion, especially large amplitude roll. Linear and cubic nonlinear roll damping coefficients are used for predicting parametric rolling and linear and squared nonlinear roll damping coefficients are used for predicting dead ship stability in the vulnerability criteria (IMO SDC 4, 2017). Linear and cubic nonlinear roll damping coefficients are adopted as shown in Eq. (44) for predicting large amplitude roll or even capsizing due to pure loss of stability and stern quartering waves.

$$
D(p)=\left(I_{x x}+J_{x x}\right)\left(\alpha \cdot p+\gamma \cdot p^{3}\right)
$$

\section{EXPERIMENTS}

The free-running experiment with a 1/40.526 scaled model of the ONR tumblehome vessel was conducted in the seakeeping basin (length: $69 \mathrm{~m}$, breadth: 46m, depth: $4 \mathrm{~m}$ ) of China Ship Scientific Research Center, which is equipped with flap wave makers at the two adjacent sides of the basin.

The ship model was used to study stability under dead ship condition (Gu et al., 2015), and here the standard bilge, rudders, and propellers are added. The principal particulars and the lines of the ONR tumblehome vessel are shown in Table 1 and Fig.2, respectively. The system parameters of the ONR tumblehome used in this standard mathematical model refer to (Umeda et al., 2016) as shown in Table 2.

Table 1 Principal particulars of the ONR tumblehome

\begin{tabular}{llc}
\hline Items & Ship & Model \\
\hline Length:L & $154.0 \mathrm{~m}$ & $3.800 \mathrm{~m}$ \\
Draft:d & $5.494 \mathrm{~m}$ & $0.136 \mathrm{~m}$ \\
Breadth:B & $18.8 \mathrm{~m}$ & $0.463 \mathrm{~m}$ \\
Depth:D & $14.5 \mathrm{~m}$ & $0.358 \mathrm{~m}$ \\
Displ.:W & $8507 \mathrm{ton}$ & $127.8 \mathrm{~kg}$ \\
$\mathrm{C}_{\mathrm{B}}$ & 0.535 & 0.535 \\
$\mathrm{GM}$ & $1.48 \mathrm{~m}$ & $0.037 \mathrm{~m}$ \\
OG & $-2.729 \mathrm{~m}$ & $-0.067 \mathrm{~m}$ \\
$L_{C B}$ & $-2.569 \mathrm{~m}$ & $-0.063 \mathrm{~m}$ \\
$\mathrm{~T}_{\varphi}$ & $14.0 \mathrm{~s}$ & $2.199 \mathrm{~s}$ \\
$\kappa_{y y}$ & $0.25 \mathrm{~L}$ & $0.25 \mathrm{~L}$ \\
$K_{z z}$ & $0.25 \mathrm{~L}$ & $0.25 \mathrm{~L}$ \\
$2 \times A_{R}$ & $2 \times 23.74 \mathrm{~m}^{2}$ & $2 \times 0.0145 \mathrm{~m}^{2}$ \\
$D_{P}$ & $5.22 \mathrm{~m}$ & $0.129 \mathrm{~m}$ \\
$\delta_{\max }$ & $35 \mathrm{degs}$ & $35 \mathrm{degs}$ \\
\hline
\end{tabular}

Table 2 System parameters of the ONR tumblehome used 


\begin{tabular}{llll}
\hline \multicolumn{5}{c}{ in this standard mathematical model } \\
\hline Items & Ship & Items & Ship \\
\hline$m_{x} / m$ & 0.015 & $1-t_{R}$ & 0.323 \\
$m_{y} / m$ & 0.680 & $1+a_{H}$ & 1.312 \\
$J_{Z Z} / I_{Z Z}$ & 0.676 & $x_{R}+a_{H} x_{H R}$ & -88.3 \\
$K_{P}$ & 3.0 & $z_{R}+a_{H} z_{H R}$ & 7.02 \\
$T_{D}$ & 0.0 & $\kappa$ & 0.647 \\
$T_{E}$ & 0.1 & $\varepsilon$ & 0.932 \\
$t_{P}$ & 0.25 & $\overline{\gamma_{R}}$ & 0.472 \\
$w_{P}$ & 0.15 & $\overline{\ell_{R}^{-}}$ & -0.709 \\
$z_{H} / d$ & 0.852 & $\Lambda$ & 1.18 \\
& & $\eta$ & 0.982 \\
\hline
\end{tabular}

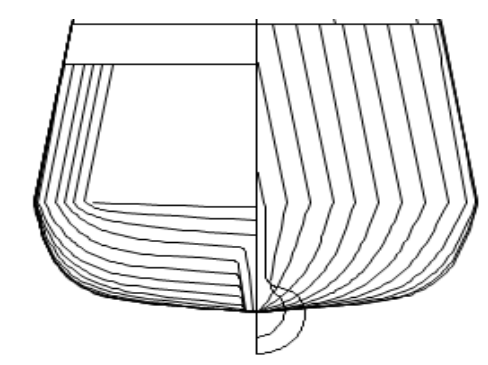

Fig.2: The ONR Tumblehome lines

The ship model was driven by twin propellers in regular following and stern quartering seas in the free-running experiment. The roll, pitch, and yaw angles were measure by the MEMS (Micro ElectroMechanical System)-based gyroscope placed on the ship model and the roll, pitch, yaw and rudder angles and the propeller rotation speed were recorded by an on-board system which is connected with an onshore control computer by wireless. The wave elevation was measured at the middle position of the basin by a servo-needle wave height sensor attached to a steel bridge which is $78 \mathrm{~m}$ in length and spans over the basin.

Free roll decay tests in calm water are conducted to obtain roll damping coefficients. The speed is a key factor for pure loss of stability. Here the nominal Froude number $(\mathrm{Fn})$ is used for the experiment of pure loss of stability in stern quartering waves by using the same specified propeller rate in calm water. The specified propeller rate corresponding to one nominal speed in calm water is determined by measuring the instantaneous position of the model ship with a total station system.

First, the model is kept near the wave maker manually by two workmen sitting on the carriage. The initial heading of the model is kept referring to the steel bridge which can rotate about its center, up to 45 degrees. Next, the wave-making system starts to generate waves. Then, the propeller revolutions increase up to the specified value according to the order received from the on-shore control computer. When the wave train propagates far enough, the model is released free near one wave crest with its initial heading, and then the model automatically runs in stern quartering waves with its specified propeller rate and autopilot course.

The ship model in the free-running experiment is shown in Fig. 3.

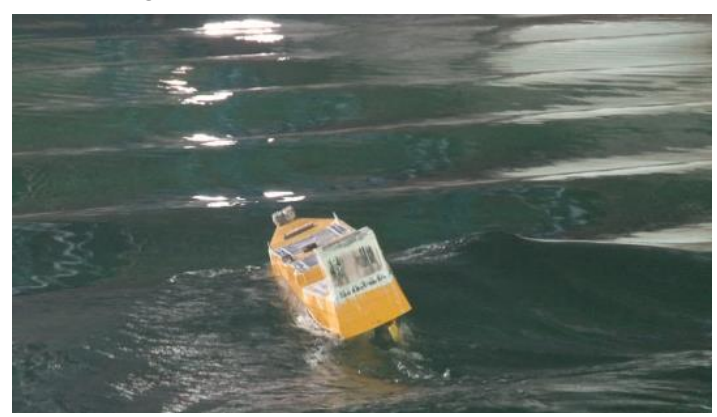

Fig.3: The ship model in the free-running experiment

\section{SIMULATIONS AND DISCUSSIONS}

\subsection{The effect of higher-order coefficients on surge motion}

The surge motion is important for predicting pure loss of stability in following seas as investigated by Lu et al. (2019). However, for ship motions in stern quartering waves, the higher-order maneuvering coefficients for hydrodynamic force acting on ship hull in the surge motion are taken into account in the MMG standard method for ship maneuvering prediction (Yasukawa \& Yoshimura, 2015), and the higher-order maneuvering coefficients without $X_{v v v v}$ are also recommended for predicting pure loss of stability by Japan (IMO SLF55，2013; Kubo et al., 2012), while these higher-order maneuvering coefficients are ignored for broaching prediction (Umeda et al., 2016). For investigating the effect of higher-order maneuvering coefficients in the surge motion on predicting pure loss of stability, the following values $X_{v w}^{\prime}=-0.1194 \quad, X_{v r}^{\prime}=-0.0649 \quad X_{r}^{\prime}=0.00841 \quad, X_{v w v}^{\prime}=0.4323 \quad$ are used based on databases of ships. 


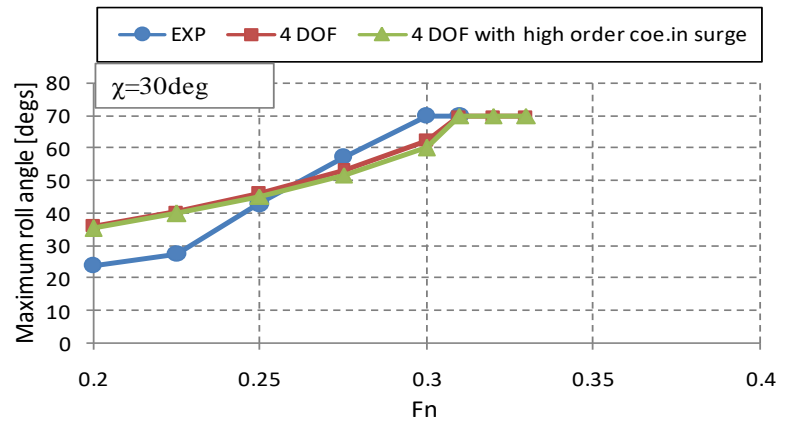

Figure 4 Comparison of maximum roll angle as a function of the Froude number between the experimental results and calculated results with the 4 DOF without and with higherorder coefficients in the surge motion with $\lambda / L p p=1.25$, $H / L p p=0.05$, and $\chi=30$ degrees.

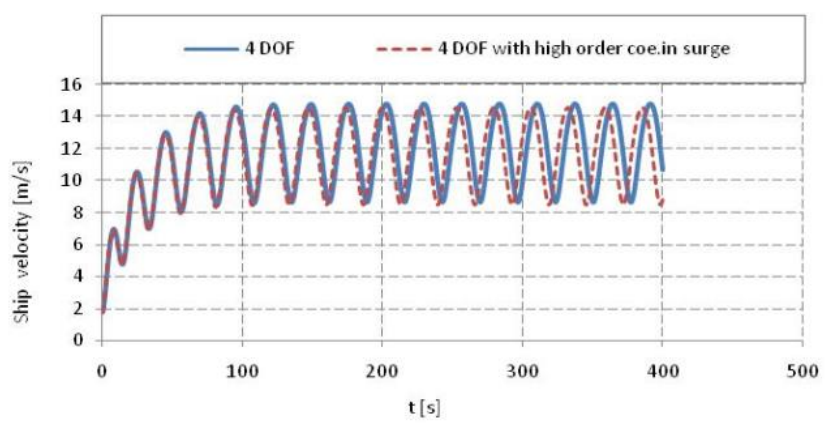

Figure 5 Comparison of ship velocity between calculated results with the 4 DOF without and with higher-order coefficients in the surge motion with $F n=0.32 / p p=1.25$, $H / L p p=0.05$, and $\chi=30$ degrees.

A comparison of maximum roll angle as a function of the Froude number between the experimental results and calculated results with 4 DOF, without and with the higher-order coefficients in the surge motion of $\lambda / L p p=1.25, H / L p p=0.05$, and $\chi=30$ degrees is carried out as shown in Fig.4. The results indicate that the effect of the higherorder maneuvering coefficients in the surge motion on predicting pure loss of stability is very small because the effect of the higher-order maneuvering coefficients on the surge motion is generally small as shown in Fig.5. The higher-order maneuvering coefficients in the surge motion are ignored in the following simulations.

\subsection{The effect of waves on roll restoring variation}

When the midship section is located on the crest in astern seas, the metacentric height is reduced and may become negative. The righting arm in calm water GZ and the restoring variations in waves with static balance method based on Froude-Krylov assumption GZw_FK are shown in Fig. 6. The stability loss at the crest is heavy, and if the state of stability loss at the crest exists long enough, capsizing could happen.

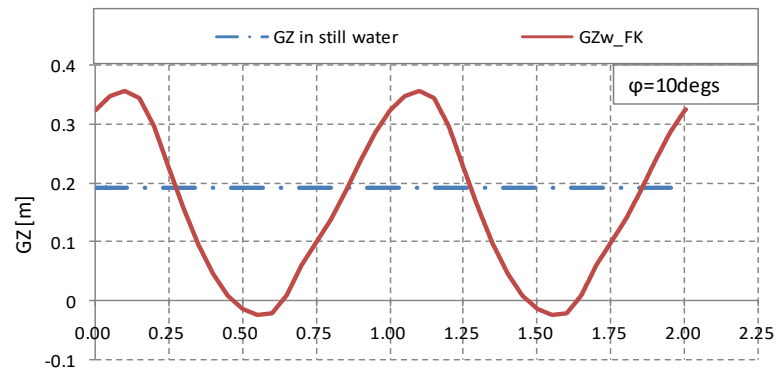

$\xi \sigma / \lambda$

Figure 6: Restoring variation in following seas with $\varphi=10$ degrees, $\lambda / L p=1.25, H / L p p=0.05$, and $\chi=30$ degrees.

\subsection{The effect of surge motion on roll restoring variation}

The nominal velocity of the ship with $F n=0.3$, the actual velocity of the ship, the wave velocity and the wave velocity considering the ship heading are shown in Fig. 7. The nominal velocity of the ship is much smaller than the wave velocity, and the maximum actual velocity of the ship is also smaller than the wave velocity. The forward speed is varied in a large range around the nominal speed of ship due to the surge motion, and the state at the crest exists longer than that at the trough.

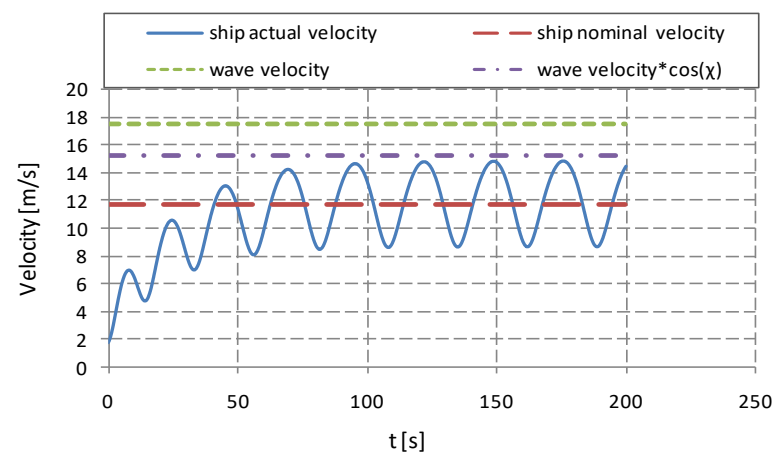

Figure 7: Comparison between ship velocity and wave velocity with nominal $F n=0.3, \lambda / L p p=1.25, H / L p p=0.05$, and $\chi=30$ degrees.

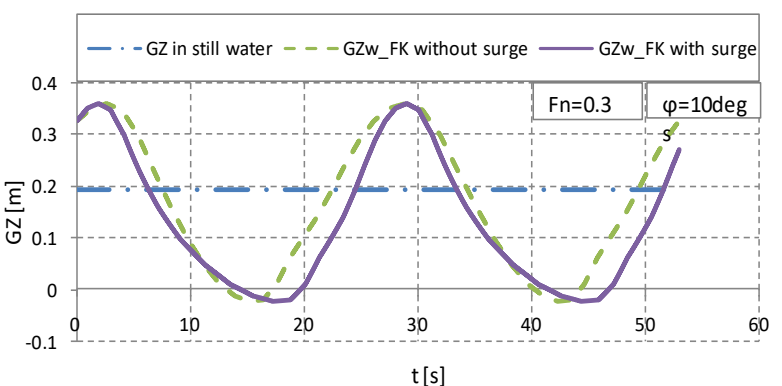

Figure 8: The effect of surge motion on restoring variation with $\varphi=10$ degrees, $\lambda / p p=1.25, H / L p p=0.05$, and $\chi=30$ degrees. 
The righting arm in calm water GZ, the restoring variations in waves based on Froude-Krylov assumption GZw_FK with the surge and without the surge in stern quartering waves are shown in Fig. 8. The state of stability loss at the crest exists longer than that at the trough because the surge motion causes the state at the crest to exist longer than that at the trough as shown in Fig. 7.

\subsection{The contribution of each element to the rolling moment}

Pure loss of stability could not pure in stern quartering waves (Kubo et al., 2012; IMO SLF55, 2013). There are other forces in the roll direction that also have some contribution to the rolling moment and the contribution of each element in the Eq. (6) to the roll moment are shown in Fig. 9 by comparing with the restoring variations in waves based on Froude-Krylov assumption GZw_FK.

The diffraction force in roll direction has a small negative contribution to the roll moment as shown in Fig. 9(a). The rudder force in the roll direction has a significant negative contribution to the roll moment as shown in Fig. 9(b) that could result in a large roll motion. The centrifugal force in the roll direction due to the added mass in the surge direction is generally small as shown in Fig. 9(c), while that due to the added mass in the sway direction is not small as shown in Fig. 9(d). The centrifugal force in the roll direction due to the maneuvering motions in the sway and yaw direction is not small as shown in Figs. 9(e) and 9(d). However, the contribution from the sway motion is negative while that from the yaw motion is positive. The heel-induced hydrodynamics force for large heel roll in calm water has a small negative contribution to the roll moment as shown in Fig. $9(\mathrm{~g})$, while the contribution to the roll moment from the higher-order maneuvering coefficients in the roll motion is also small as shown in Fig. (h).

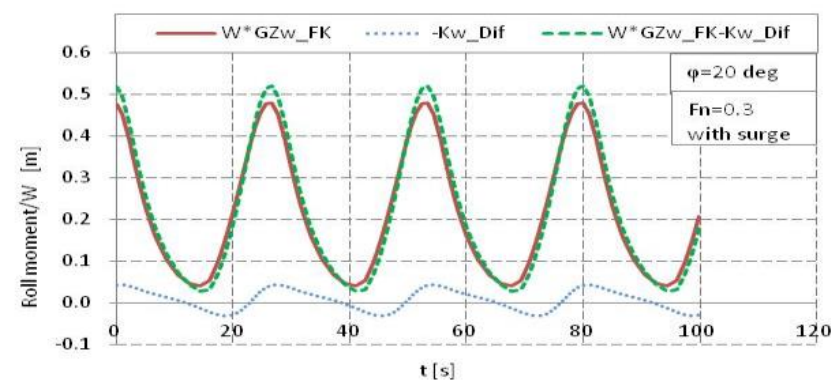

(a): The contribution to the rolling moments from the diffraction force in the roll direction.

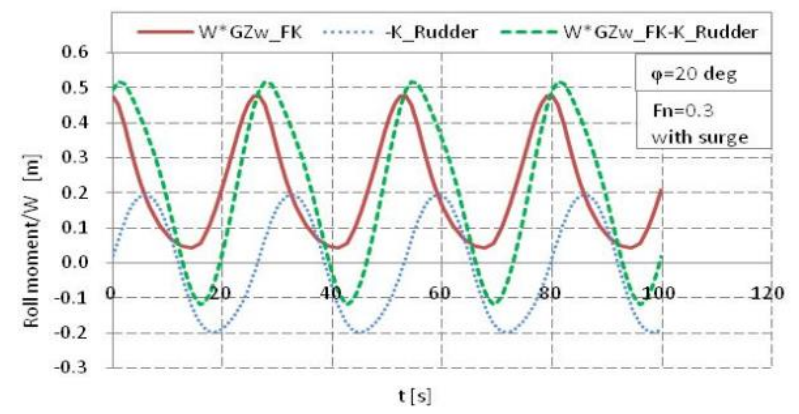

(b): The contribution to the rolling moments from the rudder force in the roll direction.

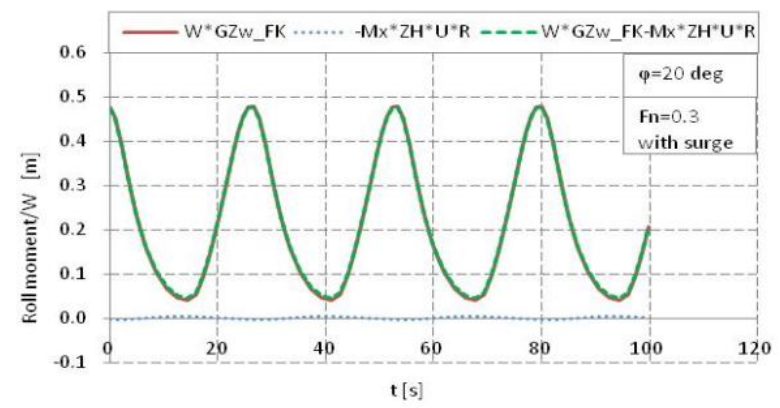

(c): The contribution to the rolling moments from the centrifugal force in the roll direction due to added mass in the surge direction.

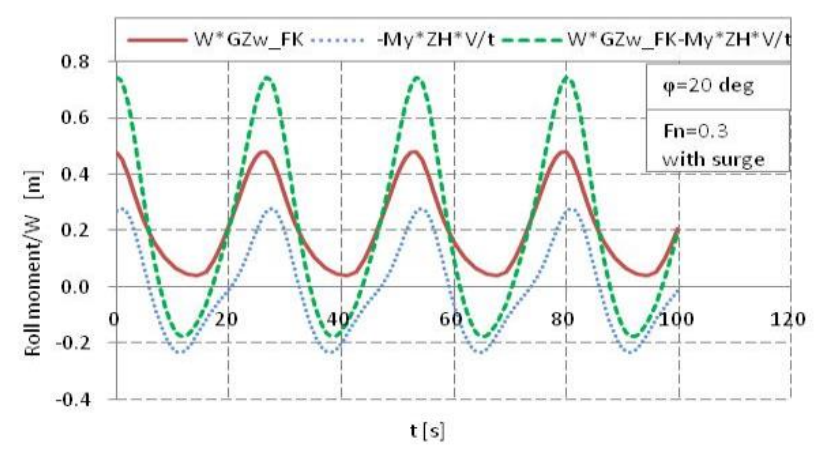

(d): The contribution to the rolling moments from the centrifugal force in the roll direction due to added mass in the sway direction.

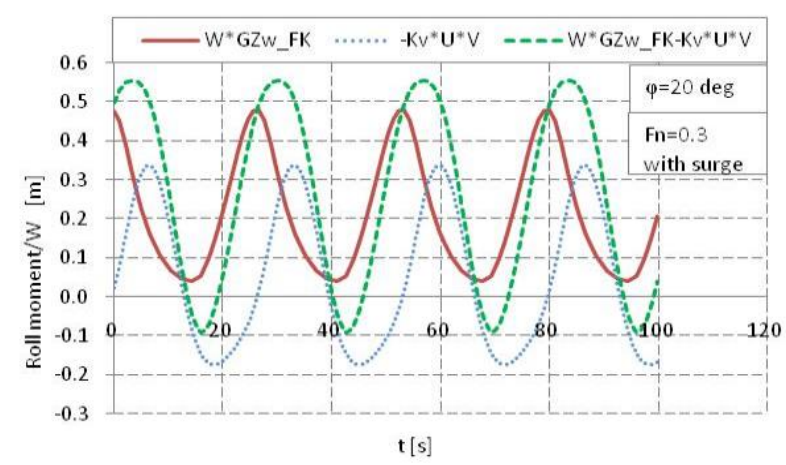

(e): The contribution to the rolling moments from the centrifugal force in the roll direction due to maneuvering motions in the sway direction. 


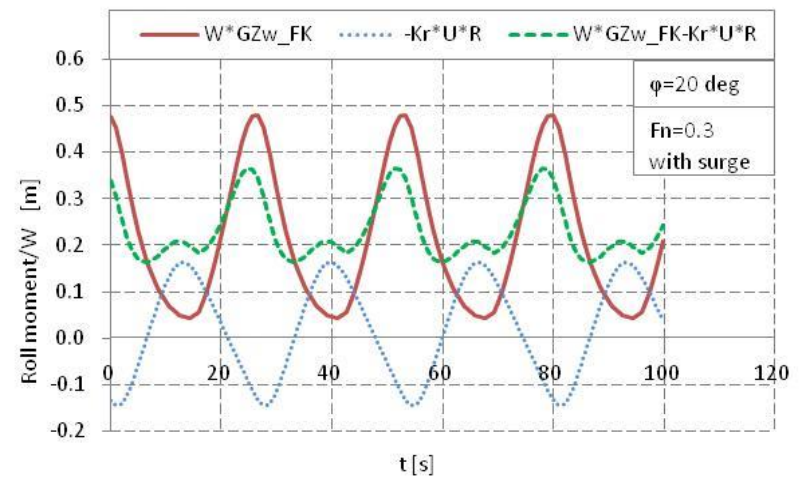

(f): The contribution to the rolling moments from the centrifugal force in the roll direction due to maneuvering motions in the yaw direction.

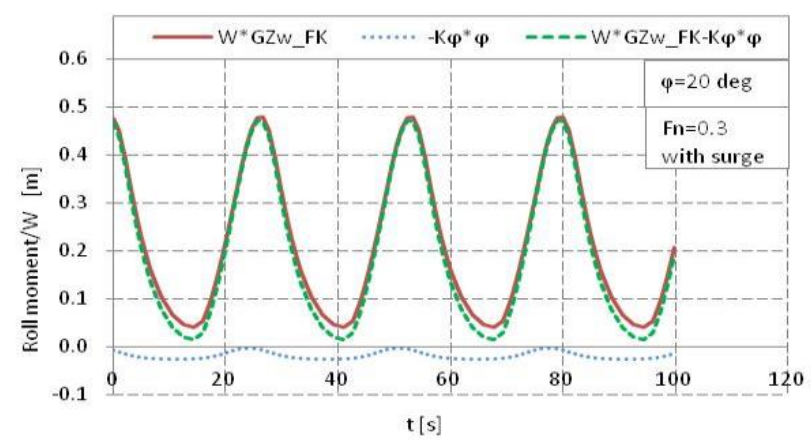

(g): The contribution to the rolling moments from the heelinduced hydrodynamic forces for large heel angles in calm water.

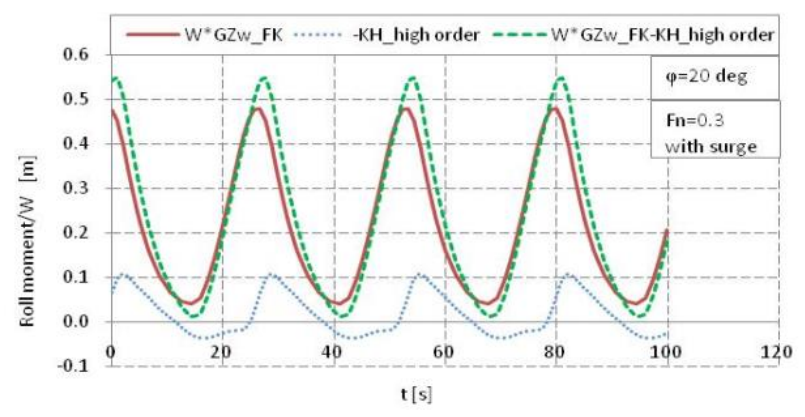

(h): The contribution to the rolling moment from the centrifugal force in the roll direction due to the higher-order maneuvering coefficients in the roll motion.

Figure 9: Comparison of the contribution to the rolling moments between the restoring variation and each force in the roll direction with $\varphi=20$ degrees, $F n=0.3$, $\lambda / L p p=1.25$, $H / L p p=0.05$ and $\chi=30$ degrees.

\subsection{The effect of the diffraction forces}

The diffraction forces, as a part of the exciting wave forces, are very important for predicting ship motions in waves. For investigating the effect of diffraction forces on predicting pure loss of stability in stern quartering waves, the simulations with and without the diffraction forces, and only without the roll motion diffraction forces are carried out as shown in Fig.10.
The mathematical model of 4 DOF only, without diffraction forces in the roll motion could predict the roll angles due to its small negative contribution to the roll moment as shown in Fig. 9(a). However, the mathematical model of 4 DOF without diffraction forces underestimates the roll angle and fails to correctly predict the capsizing range of critical ship speeds due to indirectly reducing the effect of the sway and yaw motions on the roll. This means the diffraction forces should be taken into account for predicting pure loss of stability in stern quartering waves.

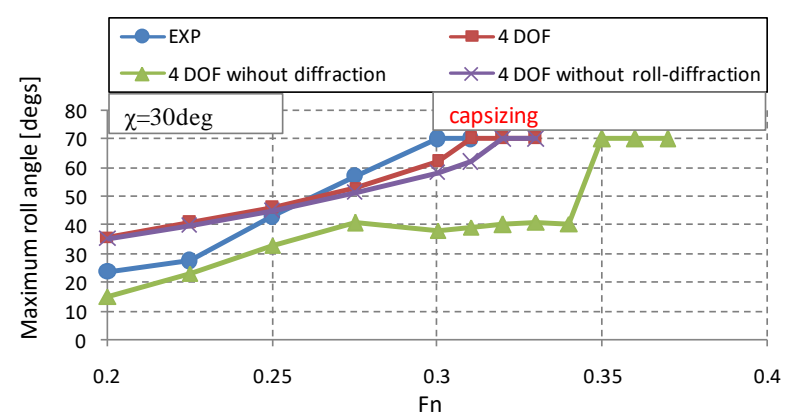

Figure 10: Comparison of maximum roll angle as a function of the Froude number between the experimental results and calculated results with the 4 DOF with and without diffraction force and only without diffraction force in the roll motion with $\lambda L p p=1.25, H / L p p=0.05$, and $\chi=30$ degrees.

\subsection{The effect of the rudder forces in the roll direction}

The rudder force is an important element in the 4 DOF mathematical model for predicting pure loss of stability in stern quartering waves, and the 4 DOF mathematical model without the rudder force in the roll direction underestimates the roll angle and fails to correctly predict capsizing range of critical ship speeds as shown in Fig. 11 due to its significant negative contribution to the roll moment as shown in Fig. 9(b).

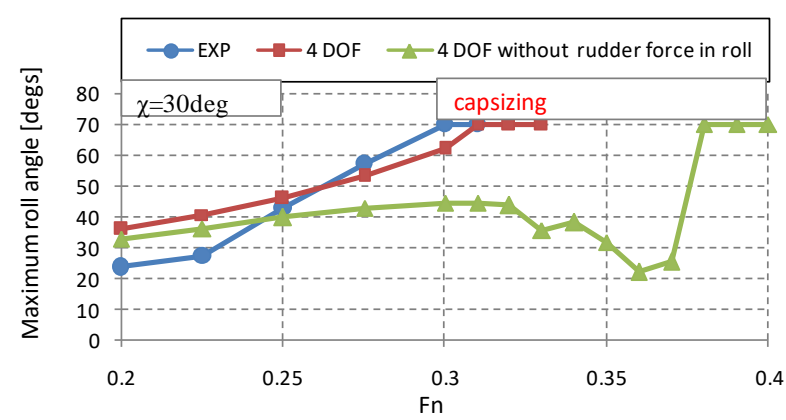

Figure 11: Comparison of maximum roll angle as a function of the Froude number between the experimental results and calculated results with the 4 DOF with and without the rudder force in the roll direction with $\lambda / L p p=1.25$, $H / L p p=0.05$, and $\chi=30$ degrees. 
5.7 The effect of the centrifugal force in the roll direction due to the added mass in the surge and sway directions

The centrifugal forces in the roll direction due to the added mass in the surge and sway directions are in investigated as shown in Figs. 12 and 13, respectively. The effect of the centrifugal forces in the roll direction due to the added mass in the surge direction is generally small due to its small contribution to the roll moment as shown in Fig. 9(c). The effect of the centrifugal forces in the roll direction due to the added mass in the sway direction is not large because its positive and negative contributions to the roll moment are near same as shown in Fig. 9(d).

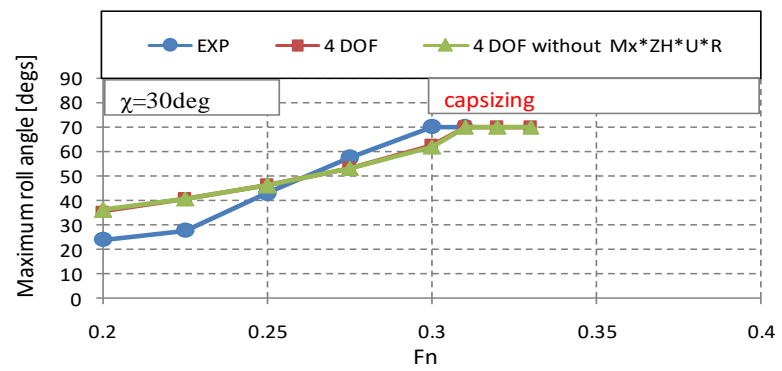

Figure 12: Comparison of maximum roll angle as a function of the Froude number between the experimental results and calculated results with the 4 DOF with and without centrifugal force in roll direction due to added mass in the surge direction with $\lambda / L p p=1.25, H / L p p=0.05$, and $\chi=30$ degrees.

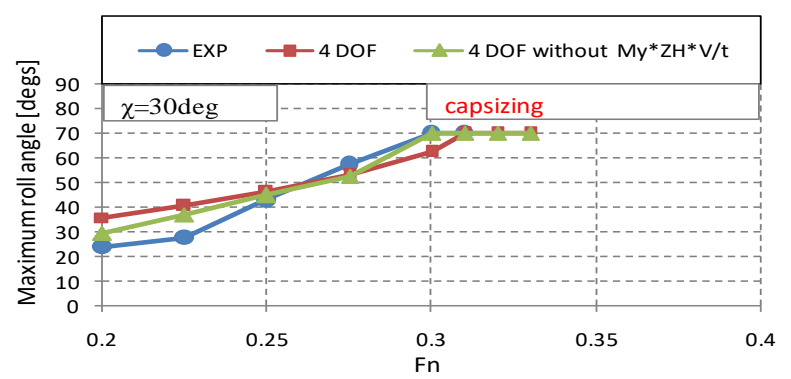

Figure 13: Comparison of maximum roll angle as a function of the Froude number between the experimental results and calculated results with the 4 DOF with and without centrifugal force in roll direction due to added mass in the sway direction with $\lambda / L p p=1.25, H / L p p=0.05$, and $\chi=30$ degrees.

\subsection{The effect of the centrifugal forces in the roll direction due to the maneuvering forces in the sway and yaw directions}

The 4 DOF mathematical without the centrifugal forces in the roll direction due to the maneuvering force in the sway direction underestimates roll angels and fails to fails to correctly predict capsizing range of critical ship speeds as shown in Fig. 14 due to its negative contributions to the roll moment as shown in Fig. 9(e).

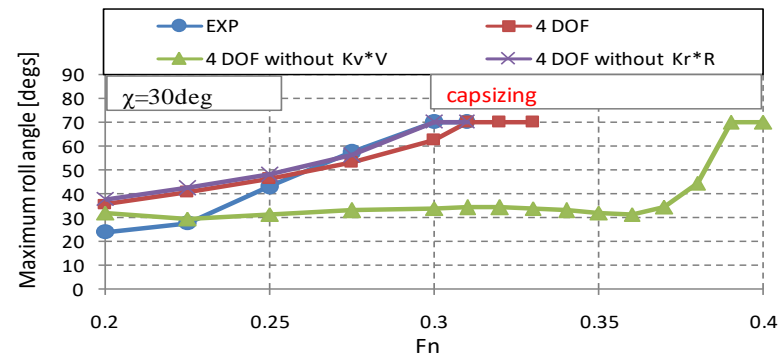

Figure 14: Comparison of maximum roll angle as a function of the Froude number between the experimental results and calculated results with the 4 DOF with and without centrifugal force in roll direction due to the maneuvering forces in the sway and yaw directions with $\lambda / L p p=1.25$, $H / L p p=0.05$, and $\chi=30$ degrees.

The calculated results with the centrifugal forces in the roll direction due to the maneuvering force in the yaw direction is larger than without that as shown in Fig. 14 due to its positive contribution to the roll moment as shown in Fig. 9(f).

\subsection{The effect of heel-induced hydrodynamic forces for large heel angle in calm water}

Pure loss of stability is accompanied by a large rolling motion. The heel-induced hydrodynamic forces for large heel angles in calm water, which are hydrodynamic lift due to underwater asymmetry induced by heel angle with forwarding velocity, could affect the prediction of pure loss of stability. The linear heel-induced hydrodynamic forces in calm water are investigated as shown in Fig.17. The 4 DOF mathematical without the linear heel-induced hydrodynamic forces, such as $Y_{\varphi}^{\prime} \cdot \varphi, N_{\varphi}^{\prime} \cdot \varphi, K_{\varphi}^{\prime} \cdot \varphi$, fails to correctly predict capsizing range of critical ship speeds as shown in Fig. 15 due to its negative contributions to the roll moment as shown in Fig. $9(\mathrm{~g})$.

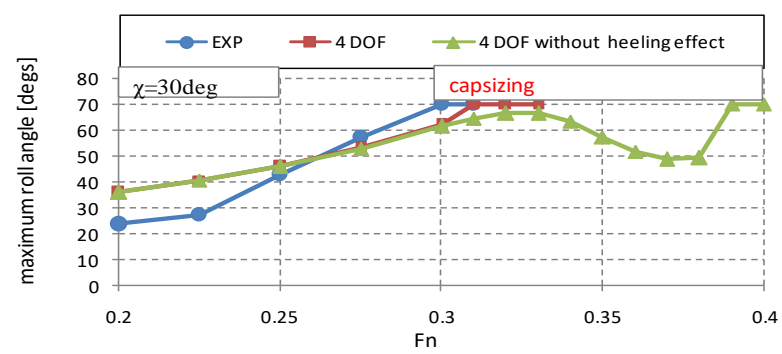

Figure 15: Comparison of maximum roll angle as a function of the Froude number between the experimental results and calculated results with the $4 \mathrm{DOF}$ with and without linear heeling effect with $\lambda L p p=1.25, H / L p p=0.05$, and $\chi=30$ degrees. 


\subsection{The effect of higher-order maneuvering coefficients on pure loss of stability}

The higher-order maneuvering coefficients of heel-induced hydrodynamic forces are not considered in this study due to the lack of referenced databases of ships. The other maneuvering coefficients mentioned in the references (Hashimoto et al., 2011b; Umeda et al., 2016) are used in this study.

The higher-order maneuvering coefficients in sway, roll and yaw motions for hydrodynamic force acting on ship hull could affect predicting pure loss of stability, and a comparison of maximum roll angle between the experimental results and calculated results with 4 DOF with and without higher-order coefficients in sway, roll and yaw motions under the condition of $\lambda / L p p=1.25, H / L p p=0.05$, and $\chi=30$ degrees are carried out as shown in Fig.16. The results indicate that the higher-order maneuvering coefficients in sway, roll and yaw motions on pure loss of stability are small due to its small contribution to the roll moment as shown $n$ Fig. 9(h).

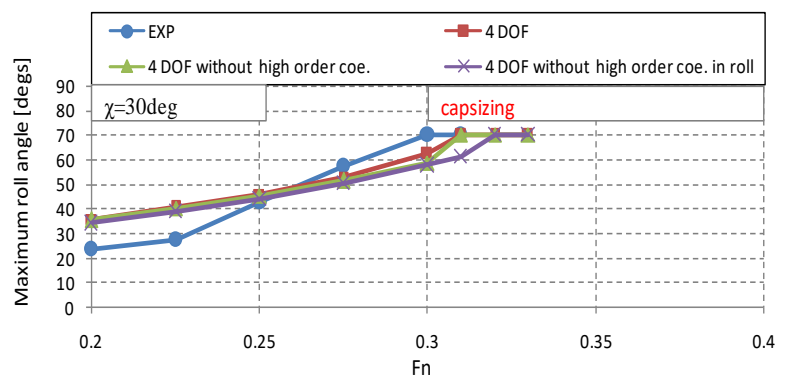

Figure 16: Comparison of maximum roll angle as a function of the Froude number between the experimental results and calculated results with the $4 \mathrm{DOF}$ with and without higherorder maneuvering coefficients in roll motions with $\lambda / L p=1.25, H / L p p=0.05$, and $\chi=30$ degrees.

\subsection{The effect of different DOF}

For investigating the effect of maneuvering motions on predicting pure loss of stability, a comparison of maximum roll angle as a function of the Froude number between mathematical models with different DOF is conducted.

As shown in Fig. 17, the mathematical models with all 3 DOF and 2 DOF coupled motion could underestimate the roll angle in stern quartering waves.

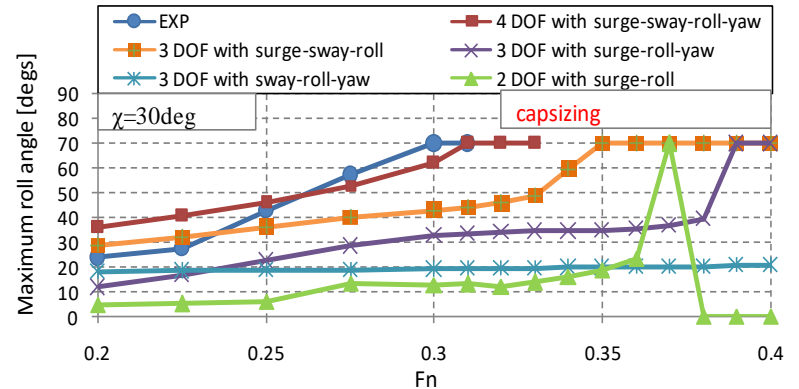

Figure 17: Comparison of maximum roll angle as a function of the Froude number between the experimental results and calculated results with mathematical models of different DOF with $\lambda / L p p=1.25, H / L p p=0.05$, and $\chi=30$ degrees

The mathematical model with 3 DOF of swayroll-yaw coupled motion not only underestimates the roll angle but also fails to predict capsizing at critical ship speeds because the state at the crest exists longer than that at the trough due to the surge motion as shown in Fig. 7 and then the state of stability loss and the negative contribution to the roll moment at the crest exist long enough. This means the surge motion is very important for predicting pure loss of stability in stern quartering waves. The surge motion cannot be ignored in the mathematical model for predicting pure loss of stability. The $2 \mathrm{DOF}$ and other 3 DOF mathematical models also fail to correctly predict capsizing at critical ship speeds because the centrifugal forces in the roll direction due to the maneuvering motions of sway and yaw have significant contributions to roll moment as shown in Fig. 9.

The mathematical model with 4 DOF of surgeroll-sway-yaw coupled motions could predict roll angle and appropriately estimate capsizing due to pure loss of stability, and pure loss of stability in stern quartering waves could not be "pure". This also supports the conclusion in Kubo et al. (2012) that the centrifugal force due to sway and yaw motions, other than the restoring reduction on a wave crest, is indispensable for explaining "pure" loss of stability on a wave crest. Therefore, both the sway and yaw motions should be considered in the mathematical model for predicting pure loss of stability in sternquartering waves.

\subsection{The type of roll motions during pure loss of stability}

The experimental results of yaw, roll, pitch motions and rudder angle in stern quartering waves are shown in Fig.18. 


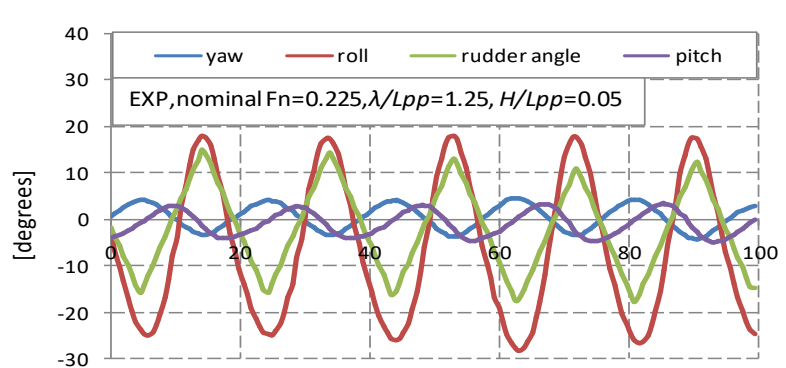

$t[s]$

(a) stable roll motion far from the critical speed of pure loss of stability

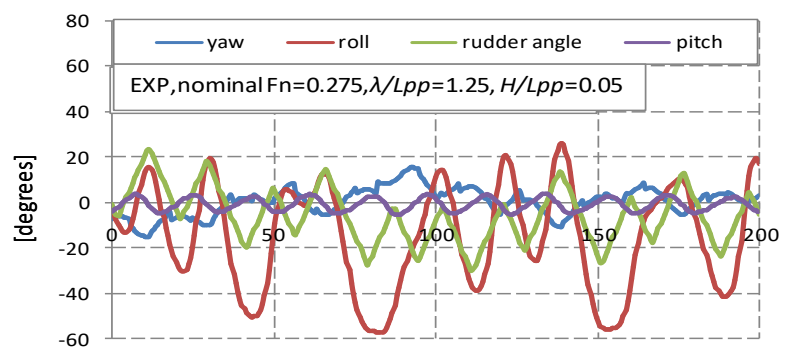

$\mathrm{t}[\mathrm{s}]$

(b) unstable roll motion near the critical speed of pure loss of stability

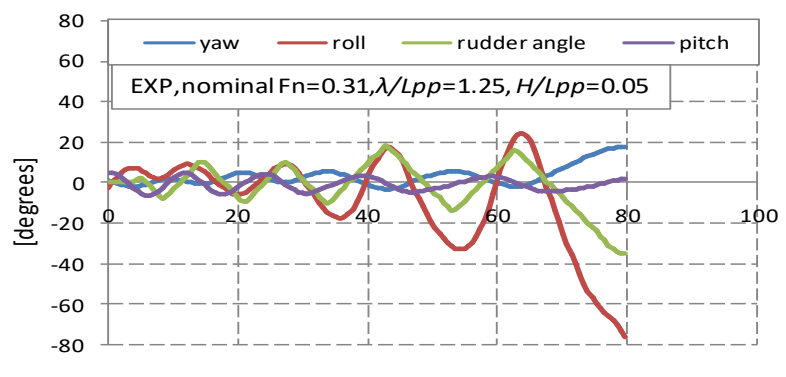

$\mathrm{t}[\mathrm{s}]$

(c) capsizing due to coupled pure loss of stability and broaching

Figure 18: Yaw, roll, pitch motions and rudder angle in the free-running experiment with $\lambda / L p p=1.25, H / L p p=0.05$, and $\chi=30$ degrees.

A stable periodic roll motion can be found when the ship speed is far from the critical speed of pure loss of stability as shown in Fig. 18(a), while an unstable roll motion can be found when the ship speed is close to the critical speed, as shown in Fig. 18(b). The capsizing due to pure loss of stability is shown in Fig.18(c), and the yaw angle reaches 20 degrees and the rudder angle reaches the maximum 35 degrees when the ship capsizes. The capsizing occurs due to pure loss of stability before developing the larger yaw angle, that is to say, the maximum rudder angle cannot correct the course and obviously broaching stops due to the capsized of the model. This could be a new phenomenon of capsizing due to coupled pure loss of stability and broaching.
We may also notice that the roll period in Fig. 18 (a) is equal to the encounter period, and it is parametric rolling whose period is equal to the encounter wave period. We can find that the second and the third roll periods in Fig. 18 (c) are also near the same with the natural roll period which could be synchronous rolling, and the fourth roll period becomes larger but is smaller than their encounter wave periods, and then capsizing occurs, and this may be also a resonant phenomenon in stern quartering waves. Capsizing in astern waves is very complicated, and pure loss of stability is only one of the reasons.

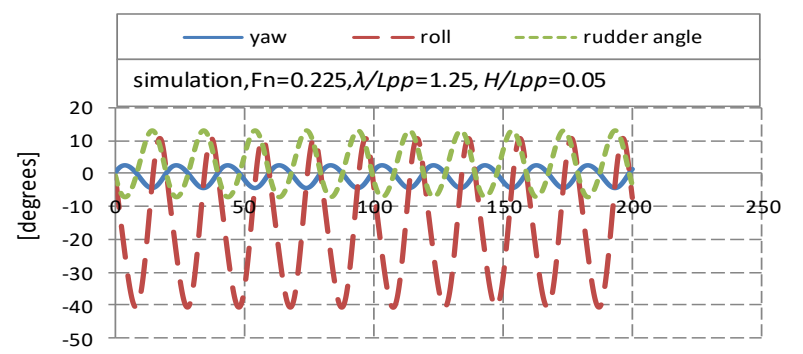

$t[s]$

(a) stable periodic roll motion far from the critical speed of pure loss of stability

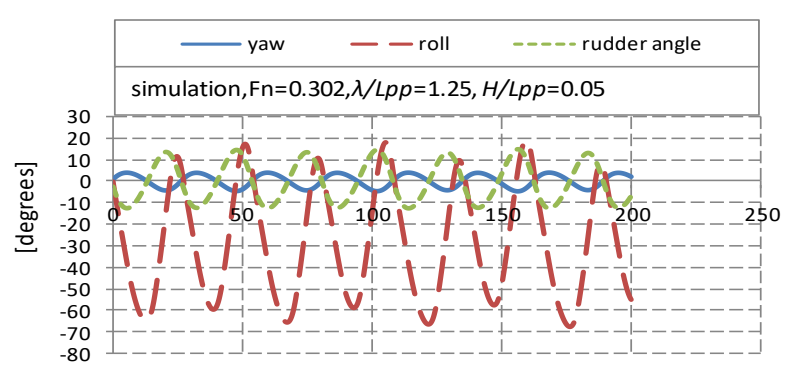

$\mathrm{t}[\mathrm{s}]$

(b) unstable roll motion near the critical speed of pure loss of stability

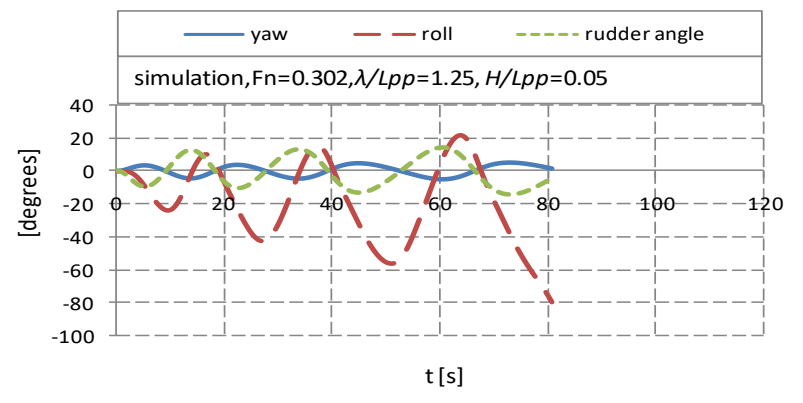

(c) capsizing due to pure loss of stability

Figure 19: Yaw, roll motions and rudder angle in the simulation with the 4 DOF mathematical model with $2 / L p p=1.25, H / L p p=0.05$, and $\chi=30$ degrees.

The calculated results of yaw, roll motions and rudder angle in stern-quartering waves are shown in Fig. 19. A stable periodic roll motion when the ship speed is far from the critical speed of pure loss of 
stability, an unstable roll motion when the ship speed is close to the critical speed and the capsizing due to pure loss of stability also can be found in the simulations with the 4 DOF mathematical model as shown in Fig. 19 (a), (b) and (c), respectively.

However, the new phenomenon of capsizing due to coupled pure loss of stability and broaching in the experiment cannot be captured by the simulations at this stage, while the unstable roll motion also cannot be completely repeated in the simulations. It appears that pure loss of stability in stern quartering waves is more complicated than our previous understanding, although the roll angle and capsizing due to pure loss of stability can be predicted accurately enough.

\section{CONCLUSIONS}

Based on the experimental and numerical study on a mathematical model of pure loss of stability with the ONR tumblehome vessel, the following remarks can be made:

1) The effect of surge motion with varied forward speed on pure loss of stability in stern quartering waves should be considered while the higher-order maneuvering coefficients in the surge motion can be ignored.

2)The diffraction forces, the centrifugal force due to sway and yaw motions with higher-order maneuvering coefficients, the heel-induced hydrodynamics force for large heel roll in calm water and the rudder force have contributions to roll moment and should be taken into account for pure loss of stability in stern quartering waves.

3) Pure loss of stability is not pure in stern quartering waves, and the 4 DOF coupled motion reestablished in this paper with the centrifugal force due to sway and yaw motions can be utilized for direct stability assessment of pure loss of stability in stern quartering waves.

As parametric roll, resonant roll, unstable roll motions and capsizing due to coupled pure loss of stability and broaching could exist during pure loss of stability. Further research will be conducted in the future to shed some light on this phenomenon.

\section{Acknowledgments}

Some contents used in this research were once guided by Prof. Naoya Umeda during the first author's Ph.D course at Osaka University supported by China Scholarship Council [No.2008606031]. The research is supported by Ministry of Industry and Information Technology of China (No. [2016] 25, 26; [2017] 614). Some contents of this paper were presented at the 13th International Conference on the Stability of Ships and Ocean Vehicles, 2018, Japan with the reviewers' useful comments. This paper is finished during the first author's visiting at University of Strathclyde supported by China Scholarship Council [No. 201905280001]. These supports are gratefully acknowledged. Dr. Boulougouris' work was supported from DNVGL and RCCL, sponsors of the MSRC. The opinions expressed herein are those of the authors and do not reflect the views of DNVGL and RCCL.

\section{REFERENCES}

Bassler, C, Campbell, B, W and McCue, L., 2007, "Dynamic Stability of Flare and Tumblehome Hull Forms in Waves", 9th International Ship Stability Workshop, CD.

Bassler C., Belenky V., Bulian G., Francescutto A, Spyrou K, and Umeda N., 2011, "Review of Available Methods for Application to Second Level Vulnerability Criteria", Contemporary Ideas on Ship Stability and Capsizing in Waves, pp 3-23.

Belenky V., Weems K., Lin W-M., 2015, "Splittime method for estimation of probability of capsizing caused by pure loss of stability", Proceedings of the 12th International Conference on Stability of Ships and Ocean Vehicles. Glasgow, UK.

Bulian G., Francescutto A., 2006, "On the effect of stochastic variations of restoring moment in longcrested irregular longitudinal sea", Proceeding 9th International Conference on Stability of Ships and Ocean Vehicle. Rio de Janeiro, Brazil. 1:131-146.

Bulian G., Francescutto A., Maccari A., 2008, "Possible simplified mathematical models for roll motion in the development of performance-based intact stability criteria-extended and revised version", University of Trieste.

Dunwoody AB., 1989, "Roll of a ship in Astern Seas-metacentric height spectra", Journal of Ship Research, 33(03):221-228.

Galbraith, A. and Boulougouris, E., "Parametric Rolling of the Tumblehome Hull using CFD", Proc. of the 12th Int. Conf. on the Stability of Ships and Ocean Vehicles (STAB2015), 14-19 June, 2015, Glasgow, UK, pp. 535-543. 
Gu M., Lu J. and Wang T.H., 2015, "Stability of a Tumblehome Hull under Dead Ship Condition", Journal of Hydrodynamics, Vol. 27 (3), pp. 452-457.

Hamamto M., and Nomoto K., 1982, "Transverse Stability of Ships in a Following Sea", Proceeding of the 2nd International Conference on Stability of Ships and Ocean Vehicles, 215-224.

Hamamoto M., Kim Y.S., 1993, “A New Coordinate System and the Equations Describing Manovering Motion of a Ship in Waves", Journal of the Society of Naval Architects of Japan, Vol. 173, pp. 209-220.

Hashimoto H., Umeda N. and Matsuda A., 2004, "Importance of Several Nonlinear Factors on Broaching Prediction", Journal of Marine Science and Technology, Vol. 9, pp. 80-93.

Hashimoto H., Umeda N. and Matsuda A.,2011a, "Model Experiment on Heel-Induced Hydrodynamics Force in Waves for Realising Quantitative Prediction of Broaching", M.A.S. Neves et al. (eds.), Contemporary Ideas on Ship Stability and Capsizing in Waves, Fluid Mechanics and Its Application 96, pp. 379-397.

Hashimoto H., Umeda N. and Matsuda A., 2011b, "Broaching Prediction of a Wave-piercing Tumblehome Vessel with Twin screws and Twin Rudders", Journal of Marine Science and Technology, Vol. 16, pp. 448-461.

IMO2013, Development of Second Generation Intact Stability Criteria, SLF 55/INF.15. Annex 12.

IMO2014, Development of Second Generation Intact Stability Criteria, Vulnerability assessment for dead-ship stability failure mode, SDC1 /INF.6.

IMO2016, Finalization Second Generation Intact Stability Criteria, SDC 3/WP.5. Annex 3

IMO2017, Finalization Second Generation Intact Stability Criteria, SDC4/WP.4.

Khanfir S., Hasegawa K., Nagarajan V., Shouji K. and Lee S.K., 2011, "Maneuvering Characteristics of Twin-rudder systems: Rudder-hull Interaction Effect on the Maneuverability of Twinrudder Ships", Journal of Marine Science and Technology, Vol. 16, pp. 472-490.

Kubo H., Umeda N., Yamane K., Matsuda A., 2012, "Pure Loss of Stability in Astern Seas -Is It Really Pure?", Proceedings of the 6th Asia-Pacific Workshop on Marine Hydrodynamics, pp. 307-312.
Kuo C., Vassalos D., Alexander JG., 1986, "Incorporating theoretical advances in usable ship stability criteria", RINA Int conf of SAFESHIP project. Ship Stab and Saf. London.

Lu J., Gu M. and Umeda N., 2017, "Experimental and Numerical Study on Several Crucial Elements for Predicting Parametric Roll in Regular Head Seas", Journal of Marine Science and Technology, Vol. 22, pp. 25-37.

$\mathrm{Lu}$ J., Gu M., 2017, "Study on Standard Mathematical Model of Pure Loss of Stability in Stern-quartering Waves", 16th International Ship Stability Workshop,5-7June 2017, Belgrade, Serbia.

Lu J., Gu M., 2019, "Model Experiments and Direct Stability Assessments on Pure Loss of Stability of the ONR Tumblehome in Following Seas", 17th International Ship Stability Workshop, 10-12 June 2019, Helsinki, Finland.

Lu J., Gu M., Wang TH., Shi C., 2018, "S Experimental and Numerical Study on Standard Mathematical Model for Pure Loss of Stability", Proceeding of the 13th International Conference on Stability of Ships and Ocean Vehicles, 16-21 September 2018, Kobe, Japan

Lu J., Gu M. and Boulougouris E, 2019, "Model Experiments and Direct Stability Assessments on Pure Loss of Stability of the ONR Tumblehome in Following Seas", Ocean Engineering (the revised version is under review)

Matsuda A. and Umeda N., 1997, "Vertical Motions of a Ship Running in Following and Quartering Seas", Naval Architecture and Ocean Engineering, No. 227, pp.47-55 (in Japanese).

Neves M., 2016, "Dynamic stability of ships in regular and irregular seas - An overview", Journal of Ocean Engineering, 120(1):362-370.

Oakley OH, Paulling JR and Wood PD, 1974, "Ship Motions and Capsizing in Astern Seas", 10th Symp Naval Hydrodynamics, pp.1-51.

Paulling JR., 1961, "The transverse stability of a ship in a longitudinal seaway", Journal of ship research, 44:37-49.

Paulling JR., Kastner S., Schaffran S., 1972, "Experimental studies of capsizing of intact ships in heavy seas". U.S. Coast Guard, Tech Rep (Also IMO Doc. STAB/7, 1973). 
Paulling JR., Oakley OH., Wood PD., 1975, "Ship capsizing in heavy seas: the correlation of theory and experiments", Proceeding of 1st International Conference on Stability of Ships and Ocean Vehicle. Glasgow.

Palmquist M., 1994, "On the statistical properties of the metacentric height of ships in following seas", Proceeding of 5th International Conference on Stability of Ships and Ocean Vehicle. Melbourne Florida.

Spyrou K.J., 1997, "Dynamic instability in quartering seas-Part III: Nonlinear effects on periodic motions", Journal of Ship Research, SNAME, Vol.41, No 3, pp.210-223.

Themelis N, Spyrou K.J., 2007, "Probabilistic assessment of ship stability trans", SNAME, 115:181-206.

Umeda N., Yamakoshi Y., 1993, "Probability of ship capsizing due to pure loss of stability in quartering seas", Nav Archit and Ocean Eng: Sel Pap Soc of Naval Arch of Japan 30:73-85.

Umeda N., Hashimoto H., Sakamoto G. and Urano S., "Research on Roll Restoring Variation in Waves", Conference Proceedings of the Kansai Society of Naval Architects, 2005

Umeda N., 1999, "Nonlinear Dynamics of Ship Capsizing due to Broaching in Following and Quartering Seas", Journal of Marine Science and Technology, Vol. 4, pp. 16-26.

Umeda N. and Hashimoto H., 2002, "Qualitative Aspects of Nonlinear Ship Motions in Following and Quartering Seas with High Forward Velocity", Journal of Marine Science and Technology, Vol. 6, pp. 111-121.

Umeda N. and Hashimoto H., 2003, "Broaching Prediction in the Light of an Enhanced Mathematical Model, with Higher-order Terms Taken into Account", Journal of Marine Science and Technology, Vol. 7, pp. 145-155.

Umeda N., Usada S., Mizumoto K., and Matsuda A., 2016, "Broaching Probability for a Ship in Irregular Stern-quartering Waves: Theoretical Prediction and Experimental Validation", Journal of Marine Science and Technology, Vol. 21, pp. 23-37.

Umeda N. and Yamakoshi Y., 1994, "Probability of Ship Capsizing due to Pure Loss of Stability in Quartering Seas", Naval Architecture and Ocean Engineering, Vol.30, pp.73-85.
Umeda N., Furukawa T., Matsuda A., and Usada S., 2014, "Rudder Normal Force during Broaching of a Ship in Stern Quartering Waves", 30th Symposium on Naval Hydrodynamics, Hobart, Tasmania, Australia, 2-7 November 2014.

Umeda N., Osugi M., Ikenaga Y., Matsuda A., 2019, "Pure loss of stability in stern quartering waves: revisited with numerical simulations reproducing accidents", 17th International Ship Stability Workshop, 10-12 June 2019, Helsinki, Finland.

Vermeer H., 1990, "Loss of stability of ships in following waves in relation to their design characteristics", Proceedings of the 4th International Conference on Stability of Ships and Ocean Vehicles, Naples.

Yasukawa H. and Yoshimura Y., 2015, "Introduction of MMG Standard Method for Ship Maneuvering Predictions", Journal of Marine Science and Technology, Vol. 20, pp. 37-52. 